\title{
Resultados de la investigación evaluativa de un curso a distancia de genética humana para profesionales de las ciencias de la salud usando como material un CD-ROM
}

(Results of the evaluative investigation of a human genetics course for health science professionals using a CD-ROM as a media)

Plácido BlanZaco

(Universidad Nacional del Litoral, Argentina)

CECILIA BRISsón

(Universidad Nacional del Litoral, Argentina)

ROBERTO RONCHI

(Universidad Nacional de Entre Ríos)

Ángela PEDRo

(Universidad Nacional del Litoral, Argentina)

RESUMEN: Introducción: La Genética ha sido la disciplina de evolución más rápida en los últimos años. En forma relativamente reciente se ha incorporado a la curricula de grado de las profesiones del área de la salud. La presente investigación busca evaluar el dictado de un curso de actualización y perfeccionamiento a distancia sobre Genética Humana usando como material didáctico el CD-ROM. Material y método: el curso fue dictado, en forma paralela a dos grupos de graduados; para uno de los grupos el material utilizado fue un CDROM y para el otro módulos impresos. Ambas modalidades incluían autoevaluaciones, heteroevaluaciones, tutorías y consultas, encuestas el curso en general (administración y materiales) y un examen final cuyo certificado fue otorgado por la Facultad de Bioquímica y Cs. Biológicas de la UNL. Resultados: los puntajes promedio obtenidos en el examen final fueron de $83,9 \%$ en los alumnos que utilizaron CD-ROM y para el otro grupo alcanza el 82,2 \%. Conclusiones: Se verifica que el sistema es idóneo para la enseñanza sin notarse ventaja a favor de una de las modalidades. En cualquiera de las formas han aprendido, lo han hecho con rendimientos muy favorables y se muestran conformes con el sistema a distancia en ambas modalidades.

Genética Humana -educación a distancia - CD-ROM - medios educativos-evaluación de la educación a distancia de posgrado -educación de posgrado en ciencias de la salud 
Plácido Blanzaco, Cecilia Brissón, Roberto Ronchi, Ángela Pedro

SUMMARY: Introduction. Genetics discipline has had a fast development during last years. Recently, it has been included in undergraduate health sciences curriculum. This study looks to evaluate a distance post graduated course in human genetics comparatively using CD-ROM or printed materials. Method: the postgraduate course has been developed in two modes: one using CD-ROM and the other using printed materials. Both modes included autoevaluation, heteroevaluation, tutorials, course evaluation (administration and materials) and a final exam. Litoral University Biochemistry School granted certifications. Results: final exams average scores have been $83,9 \%$ en CD-ROM mode and $82,2 \%$ for the other group. Conclusion: no significant differences have been identified. Both modes have shown to be effective. Postgraduate students have learnt with good scores in both systems an shown satisfied with distance system in both modes.

Human Genetics - distance education - CD-ROM - education media - post graduate distance education evaluation - post graduate health science education

\section{INTRODUCCIÓN}

Es un hecho que la Genética tuvo un desarrollo explosivo en los últimos años, tanto a nivel teórico como tecnológico. Es una herramienta poderosa y con recursos potenciales enormes para el diagnóstico, prevención y pronóstico de más de 5000 procesos que afectan al hombre. Además, la comprensión de sus bases moleculares es imprescindible para avanzar en el conocimiento de la ingeniería genética y de la biotecnología. Este avance generó un vacío curricular en la mayoría de los profesionales del área de la biología para quienes estos contenidos no fueron desarrollados en sus cursos de grado. El impacto en su práctica diaria para algunos de ellos llega desde lo teórico estrictamente hablando y para todos desde las tecnologías que directa o indirectamente utilizan la genética molecular.

\section{2. ¿POR QUÉ SELECCIONAR EL CD-ROM COMO MATERIAL?}

La experiencia de los autores de 20 años de dirigir y realizar cursos de posgrado a distancia en temas de bioquímica y medicina es que el sistema ha sido aceptado en forma casi masiva por distintos usuarios de la República Argentina y de otros países (Blanzaco P, 1998, Padula Perkins, J., 2001). En todos estos cursos, el material básico utilizado como soporte de los contenidos conceptuales y procedimentales ha sido el impreso. Varios de ellos fueron complementados con videocasetes donde se mostraron partes conflictivas de métodos o de delicada o difícil realización. Las discusiones de temas no resueltos o la resolución de casos clínicos fueron abordadas en audiocasetes cuando se evaluó que pedagógicamente era el medio más adecuado según los objetivos del aprendizaje. Abordar la Genética planteó la alternativa de incorporar nuevos recursos tecnológicos para facilitar su aprendizaje (Blanzaco P, 2001). Tópicos como la división celular, los mecanismos de las alteraciones cromosómicas estructurales y numéricas, el código genético, la manipulación del ADN, las mutaciones y el cáncer llevaron a plantear estrategias que permitan generar imágenes en movimiento y desarrollar interactividad entre el educando y los materiales de estudio disponibles. 
Resultados de la investigación evaluativa de un curso a distancia de genética humana para profesionales...

La selección del CD-ROM como soporte informático obedeció a varias razones virtualmente intrínsecas al medio: capacidad de almacenamiento; formato altamente estandarizado, esto es, su grado total de compatibilidad, lo que resulta imprescindible al momento de transmitir información; bajo precio unitario de la reproducción y de remisión a los usuarios y facilidad de transporte por los mismos con la única desventaja (considerando poseer el equipo multimedia adecuado) de la imposibilidad de escritura (son dispositivos de solo lectura).

La modalidad a distancia se basa fundamentalmente en una relación docente-alumno mediatizada. El docente está presente por intermedio de instrumentos que en sí mismos no garantizan un aprendizaje sino a través de los propósitos pedagógicos perseguidos y de la consistencia de los materiales con el resto del sistema. Esto no excluye que exista una cultura del uso de algunos medios por los usuarios, e incluso trabas sicológicas (Uñantes G, 1999) para adaptarse a un nuevo material que torne un excelente curso desde el punto de vista científico, pedagógico y técnico en un fracaso. Por esto se planificó una investigación evaluativa de un Curso de Genética Humana a nivel de perfeccionamiento y actualización para profesionales de la medicina utilizando como único material el CD-ROM frente al mismo contenido desarrollado mediante impresos.

\section{OBJETIVOS}

- Ofrecer una metodología innovadora en educación a distancia que facilite los procesos formativos intelectual - profesional a los egresados en el campo de las ciencias biológicas en el área de la genética.

- Evaluar a los usuarios del sistema con el objeto de determinar la eficacia de la nueva metodología en relación con los métodos tradicionales de cursos a distancia teniendo en cuenta la relación costo/beneficio.

- Transferir a los usuarios la necesidad de operar la metodología asociada a los multimedia.

- Actualizar en el campo de la genética el marco teórico-práctico.

\section{DESARROLLO DE LOS CURSOS}

\subsection{EL EQUIPO DE INVESTIGADORES}

El perfil de los integrantes del equipo que llevó a cabo el proceso es multidisciplinario. Incluye docentes del área de Genética de la UNL, algunos de los cuales poseen formación y experiencia en el empleo de medios educativos para la 
educación de posgrado en el área de la biología, un pedagogo y un técnico en informática. También se contó con el asesoramiento externo en lo disciplinar del Departamento de Genética de la Universidad de Navarra, España.

\subsection{EL LUGAR DE TRABAJO}

El grupo trabajó en el Departamento de Bioquímica Clínica y Cuantitativa en el aspecto concerniente a la confección y diagramación del material impreso. El material de video se obtuvo en el laboratorio del Departamento en cuanto a la faz metodológica y en ocasiones en campo cuando se trató de pacientes. La compaginación y edición del material se realizó en instalaciones del Colegio de Bioquímicos de Entre Ríos, Departamento de Educación a Distancia, donde se encuentra el equipamiento adecuado (isla de edición) y en el taller de computación del asesor en informática.

\subsection{LOS CONTENIDOS DEL CURSO}

Los contenidos teóricos y prácticos se programaron para desarrollarse en tres módulos:

1. un Módulo Básico, que incluye las unidades La Célula y el Ciclo Celular; Bases Químicas y Estructurales del Material Genético; Cromosomas Humanos, Mecanismos de Alteraciones Numéricas y Estructurales. Nomenclatura; Tipos de Herencia y Genética Poblacional

2. un módulo de Cromosomopatías y

3. un módulo de Errores Congénitos del Metabolismo.

Todos los contenidos fueron desarrollados por los profesores del Departamento de Bioquímica Clínica y Cuantitativa.

\subsection{PLANIFICACIÓN DEL CURSO SEGÚN EL MATERIAL UTILIZADO}

Si bien los objetivos planteados para el curso eran los mismos, independientemente del soporte utilizado y las evaluaciones eran las mismas desde el momento que se trata de una evaluación comparativa, el diseño, la estructuración y el desarrollo de los materiales tuvo características propias para cada modalidad.

\subsubsection{Impresos}

El contenido conceptual y procedimental fue adaptado para educación a distancia mediante material impreso. Se diseñó o adaptó específicamente material 
Resultados de la investigación evaluativa de un curso a distancia de genética humana para profesionales...

gráfico y se puso especial énfasis en destacar conclusiones parciales, generalizaciones y preguntas al estudiante que generaran reflexión sobre el tema en estudio. Cada módulo incluyó autoevaluaciones con las correspondientes respuestas sugeridas y un cuestionario de heteroevaluación para remitir.

\subsubsection{CD-ROM}

La confección del CD-ROM se desarrolló en varias etapas: una etapa a cargo de los docentes del curso en los temas específicos de genética humana, otra a cargo de estos docentes y los docentes especializados en educación a distancia y una tercera etapa a cargo de los docentes especializados en educación a distancia y los técnicos en programación.

En la primera etapa los docentes de genética humana prepararon en soporte electrónico (MS Word 8.0) el material conceptual que se emplearía tanto para el curso a distancia convencional como para el CD ROM.

En la segunda etapa se adaptaron los contenidos conceptuales para CD, buscándose el diseño general de las secuencias temáticas que el alumno debería seguir y la presentación de las mismos en las pantallas y se propusieron las instancias interactivas que facilitarían el aprendizaje. Como producto de esta etapa se obtuvo, para cada tema, una diagrama en árbol jerarquizador de los temas que permitió individualizar las conexiones secuenciales entre los mismos. Cada rama representa un archivo de texto, con las indicaciones para su presentación por pantallas y una referencia a las imágenes y situaciones interactivas propuestas para el tema.

La tercera etapa, el técnico en programación adaptó los contenidos de lo recibido de la etapa anterior al programa principal, Front Page 98 (lenguaje HTML). Este incluye en la confección lenguaje Java, Java Script y Visual Basic. Se utilizan: Corel Draw 8 para gráficos y diseños; programas animadores Gif; programas para gráficos Photoimpact; Photo Shop y Corel Photopaint de la versión 8 para videos y animaciones y sonidos con grabaciones en consolas de audio con FM y transformador a archivos con extensión wav y mid. Al final se compiló para crear un ejecutable (.exe) del inicio. Toda esta etapa en interacción permanente entre el técnico en programación y los docentes especializados en educación para obtener un producto con el máximo rendimiento para el aprendizaje: alternativas interactivas, sonidos, imágenes, videos, etc. resueltas a partir de las opciones brindadas por la informática.

El principal desafío en la diagramación del CD-ROM ha sido para nosotros superar dificultades que observamos como constante en otros materiales del mismo tipo revisado oportunamente: el seguimiento de la secuencia de estudio sin dispersión generada por las alternativas operacionales ofrecidas a través del ac- 
Plácido Blanzaco, Cecilia Brissón, Roberto Ronchi, Ángela Pedro

ceso a través de la pantalla, especialmente grave, en forma potencial, para aquellos usuarios poco entrenados en el uso de este medio.

El producto final incluye una introducción sobre el manejo del material y sobre el desarrollo del curso: contenidos, evaluaciones, tutorías, consultas, etc., los objetivos del curso y los criterios de enfoque del tema y los docentes e instituciones involucrados. En cada tema se desarrollaron los contenidos conceptuales, incluyéndose animaciones y cuestiones propuestas respondidas en audio por el profesor, con la alternativa de acceder a ellas por pantalla si no se contara con placa de sonido. Cada unidad temática tiene incluida su autoevaluación con la posibilidad directa sobre la pantalla de acceso a la respuesta sugerida y su heteroevaluación. La heteroevaluación puede ser enviada directamente desde allí por correo electrónico ya que se salvó la imposibilidad de escritura del soporte creando un acceso a Word en ese punto. También puede emplearse para comunicarse con el tutor. Si el usuario no dispusiera de correo electrónico tiene la posibilidad de imprimirse y remitirse por correo o fax. También está consignada en cada tema la bibliografía recomendada y se dispuso un glosario general de acceso directo desde cualquiera de las unidades.

\subsection{IMPLEMENTACIÓN DEL CURSO}

Se implementó una inscripción para el cursado en la que se pactó el dictado del curso con el compromiso de los usuarios de responder a las encuestas propuestas y de no abandonarlo en la medida de sus posibilidades. A cambio los alumnos recibían el curso sin cargo alguno con la posibilidad de obtener un certificado extendido por la Facultad de Bioquímica y Cs. Biológicas que aprobó los contenidos para su dictado bajo la forma de curso de actualización y perfeccionamiento previa evaluación final. Los alumnos (13) se distribuyeron aleatoriamente en dos grupos: Grupo I (6 alumnos): cuyo material de estudio consistió en módulos impresos y b) Grupo II (7 alumnos): emplearon como material de estudio un CD-ROM. Ambos grupos recibieron un instructivo acerca de los materiales de estudio y su manejo y de las características del curso y se les asignó un tutor. 
Resultados de la investigación evaluativa de un curso a distancia de genética humana para profesionales...

Tabla 1. Características de los usuarios

Alumno Sexo Domicilio e-mail F. informática F. Genética Profesión Grad. Univ. GRUPO I: material impreso

\begin{tabular}{ccccccccc}
\hline 1 & $F$ & Sta.Fe & Sí & No & No & Bioq. Dr.Qca & 1993 & UNL \\
\hline 2 & $F$ & Sta.Fe & Sí & No & No & Bioq. & 1999 & UNL \\
\hline 3 & $F$ & Sta.Fe & Sí & No & No & Bioq. & 2000 & UNL \\
\hline 4 & $M$ & Sta.Fe & No & No & No & Bioq. & 2000 & UNL \\
\hline 5 & $M$ & Paraná & No & No & No & Bioq. & 1999 & UNL \\
\hline 6 & $F$ & Gchú & Sí & No & No & Bioq. & 1984 & UNL \\
\hline Grupo II: material CD-ROM & & & & & & \\
\hline 1 & $F$ & Sta.Fe & Sí & No & No & Bioq. & 1999 & UNL \\
\hline 2 & $M$ & Paraná & Sí & No & No & Bioq. & 1999 & UNL \\
\hline 3 & $F$ & Sta.Fe & Sí & No & No & Bioq. & 1999 & UNL \\
\hline 4 & $F$ & Gchú & Sí & No & No & Bioq. & 1983 & UBA \\
\hline 5 & $M$ & Sta. Fe & Sí & No & No & Bioq. & 1999 & UNL \\
\hline 6 & F & Sta. Fe & Sí & No & No & Bioq. & 2000 & UNL \\
\hline 7 & $M$ & Sta. Fe & Sí & No & No & Bioq. & 1996 & UNL \\
\hline
\end{tabular}

Referencias: $F$. informática: se refiere a formación informática sistemática; $f$. genética: se refiere a formación en genética de posgrado o actualización y perfeccionamiento posgraduación; Grad.: año de graduación, Univ. Universidad que otorgó el título de grado; F: femenino; M: masculino; Sta.Fe: cidudad de Santa Fe; Gchú: ciudad de Gualeguaychú; Bioq. Bioquímico; Dr. en Qca.: doctor en Química; UNL: Universidad Nacional del Litoral; UBA: Universidad Nacional de Buenos Aires.

Junto al material de aprendizaje, los alumnos recibieron encuestas para la evaluación de los distintos elementos del sistema.

Respecto a las tutorías, estuvieron a cargo de los integrantes del equipo de investigación que se desempeñan como docentes en el área de genética. De esta manera operaron tanto en los aspectos generales del curso como en el asesoramiento disciplinar específico. Las consultas se realizaron por correo electrónico $\mathrm{y}$ en forma excepcional se concertaron entrevistas personales.

Las heteroevaluaciones se recibieron de acuerdo a un cronograma establecido y se reenviaron a los usuarios corregidas.

Finalizado el dictado se realizó la evaluación final. Esta tuvo carácter presencial, individual y escrita y se utilizó una misma herramienta para los dos grupos que incluyó preguntas de elección múltiple, verdadero o falso, abiertas, resolución de problemas y de casos clínicos. 
Plácido Blanzaco, Cecilia Brissón, Roberto Ronchi, Ángela Pedro

\section{RESULTADOS}

La evaluación permitió observar diversas constataciones durante la experiencia, que se comentan a continuación.

\subsection{CURSO IMPRESO}

Los alumnos debieron evaluar la redacción, el orden, la diagramación, el formato, la corrección gramatical y la estilística del material. La calificación relativa a los módulos ha sido de excelente y muy buena, con un promedio de 70,6 \%.

Las técnicas de conducción del aprendizaje se considera que siempre fueron adecuadas $(45,5 \%)$ y generalmente $(54,5 \%)$. Las mismas permitieron su participación activa siempre $(40 \%)$ y generalmente $(50 \%)$.

En cuanto al tratamiento de los contenidos, fue adecuado siempre para el $27,2 \%$ y generalmente para el $72,7 \%$.

Están conformes con la presentación de la información escrita porque fue ordenada: siempre para el $81,8 \%$ y generalmente para $18,2 \%$.

Con respecto a la comprensión, siempre fue fácil para el 27,2\%; generalmente para el $54,5 \%$ y a veces para el $27,2 \%$.

Las instrucciones (guías) para el uso del material fueron claras en un 100\% y precisas en el 66,6\% en el primer módulo; en el segundo las respectivas cifras son $60 \%$ y $50 \%$.

Se podría interpretar que en el segundo fueron más críticos o más analíticos. Pero también que la calidad fue menor. Sin embargo, se reconoce que la dificultad se debe a la naturaleza del tema: para los estudiantes eran novedad total; es decir, resultó abstracto porque carecían de conocimientos previos vinculados.

Ninguno de los participantes halló elementos distractores en el material.

En lo que se refiere a la tutoría, para el 66,6 \% el asesoramiento recibido fue muy bueno, y bueno para el 33,3\%.

El nivel o preparación académica del tutor es considerado excelente por el $83,3 \%$ y muy bueno por el $17,7 \%$.

Estiman que la calidad y adecuación de la comunicación con su tutor fue excelente en un $25 \%$ y muy buena en el $62 \%$, en ambos módulos. Sin embargo, esa comunicación fue escasa en dos tercios de los casos; el resto no se comunicó. 
Resultados de la investigación evaluativa de un curso a distancia de genética humana para profesionales...

La capacidad de trabajo del tutor es calificada de destacable por el $75 \%$ y suficiente por el resto. En todos los aspectos consultados su desempeño se considera satisfactorio y no tuvieron obstáculos con respecto a la tutoría.

Quienes se comunicaron con el tutor lo hicieron solo una vez. Fue en la mayoría de los casos para tratar la interpretación de consignas; otro lo necesitó para la interpretación de textos. No obstante, todos afirman que se les proveyó y aseguró una permanente comunicación de doble vía.

En lo referido al alumno, el 72,7 \% reconoce que siempre pudo aprender respetando su ritmo y las condiciones establecidas; generalmente pudo hacerlo el $27,2 \%$.

Los plazos otorgados para estudiar, presentar trabajos, exámenes, etc., fueron adecuados en el $100 \%$ de los casos.

La incidencia del sistema adoptado fue muy buena en la calidad de la enseñanza; el nivel de aprendizaje equivaldría a otros sistemas; y en cuanto a motivación e interés, la mayoría considera que es el mismo, pero para otros incentiva el estudio.

Con respecto a los conocimientos previos, reconocen que tenían los necesarios y que se tomaron en cuenta en el material.

Un $40 \%$ reconoce que los medios empleados incrementaron su comunicación con los responsables científicos y técnicos del curso con respecto a su experiencia en cursos presenciales. Asimismo, afirman (71,4\%) que los medios empleados incrementaron su participación activa con respecto a dicha experiencia.

En general, como fortalezas del sistema citan el material entregado, el manejo del tiempo de estudio, la flexibilidad y la interrelación.

\subsection{CURSO EN CD-ROM}

Como factor de entrada para comprender mejor algunas respuestas y sus consecuencias sobre el manejo del material, hay que señalar que los alumnos carecen de formación informática sistemática; el uso que hacen de ella es empírico. Eso conduce a que su experiencia con el CD-ROM tenga una limitada fluidez y que estas habilidades sean una interferencia (aunque probablemente leve, pero incidente) en su relación con el material.

La instalación del CD-ROM resultó sencilla (75\%), excepto en un caso.

La facilidad en el uso del sistema para buscar, retroceder, avanzar, copiar e imprimir fue diversa. Para el 66,6\% fue sencillo buscar, pero resultó complicado 
para dos personas; en cambio, para retroceder/avanzar fue sencillo para el $55 \%$ y medianamente sencillo para el 33,3\%. Se destaca la dificultad para imprimir que tuvieron tres estudiantes.

Un aspecto a destacar es la lectura de los textos. La mayoría afirma que resultó molesta por los colores empleados y por su débil y difuso contraste con el fondo. La califican de incómoda y cansadora. Sin embargo, para 2 fue sencilla, pero coinciden con respecto a los colores.

En cuanto al contenido informativo, fue calificado como actualizado (77 \%); de nivel adecuado (100\%), con estructura bien organizada (77 \%) lingüísticamente correcto $(77 \%)$, aunque dos personas hallan errores.

El contenido fue motivador para todos en el primer módulo; en el segundo solo para un participante e indiferente para dos.

En cuanto al modo de explotación del contenido, la mayoría hizo exploración previa, tal como se recomendaba. Luego siguen quienes leyeron en pantalla. Solamente uno imprimió.

Sobre las ventajas halladas en el sistema, mencionan al resumen al final de cada tema, el poco lugar que ocupa (a diferencia de lo que necesita el papel) y a la facilidad para guardarlo.

Como inconveniente con respecto al papel hallan que no se puede subrayar o marcar con color, no se puede llevar a todos lados y hay que dedicarle tiempo (el impreso se puede leer en cualquier momento).

Sobre el nivel de dificultad, lo consideran semejante a cualquier curso. En lo que se refiere a los itinerarios para estudiar, hacer las actividades y resolver los problemas, en general siguieron los recomendados en el material.

Con respecto al diseño general, todos estiman adecuados los títulos, menús, ventanas, iconos y botones, así como los espacios de texto e imagen son equilibrados para el 100\%. También los elementos multimedia (gráficos, fotografías, voz, música) son adecuados para el $71,4 \%$, aunque escasos para el $28,6 \%$.

Dicen que el estilo y lenguaje, la tipografía, el color y la composición son buenos en el $55 \%$ de los casos, pero regulares en el 44,5\%. Además, afirman que la disposición de la pantalla y su distribución es equilibrada ( $75 \%$ y armónicas (25 $\%$ ), así como la integración de medios para facilitar el aprendizaje fue lograda en general para $71,4 \%$ y a veces para dos participantes.

Los recursos para comunicarse (con el tutor, con la institución) fueron de acceso sencillo en el $100 \%$ de los casos que lo utilizaron; pero no todos se comunicaron. 
Resultados de la investigación evaluativa de un curso a distancia de genética humana para profesionales...

Las exigencias técnicas (equipo, complejidad, memoria, velocidad, conexión a redes; particularidades de manejo) estuvieron de acuerdo a las especificaciones recibidas en un $87,5 \%$. Hay un caso negativo, atribuible a destrezas previas.

Creemos muy destacable que el programa es considerado fiable en un $100 \%$.

Sobre los obstáculos en la utilización del CD-ROM, predomina el reconocimiento de «la falta de costumbre».

En lo que se refiere a la tutoría: el asesoramiento recibido es considerado entre bueno y muy bueno por quienes responden y es suficiente para todos la disponibilidad del tutor. Su nivel o preparación académica es calificada entre excelente y muy buena por todos los participantes. También estiman adecuada y de calidad la comunicación mantenida, aunque en general fue escasa y el $71 \%$ no se comunicó. Solo uno manifiesta que fue frecuente.

En lo que respecta al alumno, el 83,3\% pudo aprender respetando su ritmo y las condiciones establecidas, dentro de lo cual los plazos otorgados para estudiar, presentar trabajos, exámenes, etc., fueron adecuados en el $100 \%$ de los casos.

Los usuarios opinan que, en general, el sistema adoptado incidió favorablemente en la calidad de la enseñanza, en el nivel de aprendizaje y en su motivación e interés.

Con respecto a los conocimientos previos, quienes responden afirman que tenían los necesarios y que se tomaron en cuenta en el curso.

En lo referido a la comunicación, se manifiesta escasa utilización. Interpretamos que el sistema y el material fue el adecuado como para no necesitarla. Sin embargo, son interesantes de mencionar dos tipo de contactos registrados: uno por disidencia con el autor y otro por dudas conceptuales.

La mitad de los consultados afirma que los medios empleados incrementaron su comunicación con los responsables científicos y técnicos del curso con respecto a su experiencia en cursos presenciales, y que incrementaron su participación activa con respecto a su experiencia presencial para el 83,3\% de los casos.

En opinión de todos, la comunicación de doble vía estuvo asegurada y fue permanente.

Como fortaleza del sistema, valoran positivamente los materiales, sin que se mencione alguna debilidad.

Sintetizando, podríamos decir que en general se presentan resultados concordantes con los esperados, con algunas diferencias que han surgido de la implementación del sistema. 
Por otra parte, se ha reunido datos útiles para revisar los materiales y diversas partes del sistema, así como sobre el aspecto académico de los contenidos, especialmente relacionados con la base previa de los estudiantes en algunos temas.

Respecto a las características del CD-ROM las opiniones de los participantes presentan una importante dispersión en los 20 aspectos consultados.

Sólo concuerdan todos en que la navegación está bien estructurada; que los íconos representan claramente su propósito; y para uno hay algunos elementos distractivos en el material.

Son mayoría los que responden que: es rápido el acceso a las páginas; se perciben claramente las opciones disponibles; el diseño de las pantallas es en general claro y atractivo; se usan adecuadamente los gráficos y los colores; las imágenes son claras y comprensibles; las animaciones son adecuadas; y que el correo electrónico está incluido y bien visible en la página para comunicarse con el tutor o coordinador.

También son mayoría quienes están de acuerdo en que las páginas y los textos tiene una extensión adecuada; y que el contenido no es engorroso.

\subsection{APRENDIZAJE}

El promedio general obtenido en la evaluación final es algo superior $(83,9 \%)$ en los alumnos del sistema en CD; el otro grupo alcanza el 82,2\%.

Se observa además que tanto el rendimiento mínimo como el máximo aparecen en aquel grupo; es decir, se da una mayor dispersión de valores.

\begin{tabular}{lcc} 
& Convencional & CD-ROM \\
Promedio & 82,2 & 83,9 \\
\hline Resultado A1 & 91,4 & 89,8 \\
\hline Resultado A2 & 77,8 & 83,2 \\
\hline Resultado A3 & 77,5 & 78,8 \\
\hline
\end{tabular}

Analizando los ítems componentes de la prueba, se observa que hubo errores máximos de medio punto en 11 de los 22 que la componen. Tampoco en esto aparece alguna recurrencia significativa. Dentro del total (24 registros), 10 se presentan en quienes usaron el sistema convencional y 14 en el nuevo; todos con una gran dispersión ya que la mayor cantidad de fallas hallada para un mismo ítem es de 4. temas.

Es decir, este aspecto tampoco arroja una marcada ventaja de uno de los sis- 
Resultados de la investigación evaluativa de un curso a distancia de genética humana para profesionales...

El promedio obtenido entre los alumnos egresados antes de 1990 es menor (78.2\%) que el obtenido por los alumnos graduados posteriormente $(85.6 \%)$ y puede atribuirse a la variación curricular de las carreras de grado. Este aspecto tampoco arroja ventaja a alguno de los sistemas, si bien puede observarse un valor individual menor en un alumno graduado recientemente que cursara por impresos frente a un alumno de graduado antes de 1990 que cursara por CD-ROM.

\section{CONCLUSIONES}

Hay dos aspectos centrales logrados:

1) La elaboración de un sistema operatorio, en consonancia con el objetivo propuesto al encarar el proyecto.

Está completo y disponible. Apto para ser actualizado, mejorado y utilizado con un número indeterminado de estudiantes. Apto también para publicarse en Internet, con leves diferencias, adosándole algunos mecanismos propios de la red, principalmente para las comunicaciones. Con ello adquiere el potencial de un alcance ilimitado, al menos geográfica y numéricamente.

2) La verificación de que el sistema es idóneo para la enseñanza. Si bien no se ha constatado de modo contundente la ventaja a favor de una de las modalidades, dentro de la reducida escala de la experiencia realizada, queda visto claramente que en cualquiera de las formas propuestas los estudiantes han aprendido, lo han hecho con rendimientos muy favorables y se muestran conformes con el sistema.

Por lo tanto, los parejos resultados obtenidos serían independientes de la forma del material en sí mismo, y estarían asociados tal vez al sistema a distancia en general.

Los participantes han hallado como fortalezas del sistema el material entregado, el manejo del tiempo de estudio, la flexibilidad y la interrelación. Cuatro factores que se consideran pilares en educación a distancia.

Esta información es especialmente importante teniendo en cuenta la relación costo/beneficio de la producción, duplicación y distribución de los materiales para la realización del curso de forma masiva. 
Plácido Blanzaco, Cecilia Brissón, Roberto Ronchi, Ángela Pedro

\section{REFERENCIAS BIBLIOGRÁFICAS}

ADELL, J. (1995). La navegación hipertextual en el World-Wide-Web: implicaciones para el diseño de materiales educativos. II Congreso de Nuevas Tecnologías de la Información y Comunicación para la Educación, Palma de Mallorca.

BlanZACO, P.; BRISSÓN, C. (1998). «Conclusiones de 15 años de planificación y realización de cursos de posgrado a distancia en bioquímica. Perspectivas.» Seminario de educación a distancia para América Latina y el Caribe. Reunión preparatoria de la Conferencia Mundial de Educación a Distancia, Viena 99. Buenos Aires.

BlanZaCo, P.; Brissón, C; RonCHI, R. (2001). Diseño y evaluación de un CD-ROM para el aprendizaje de genética humana a distancia. INFOUNI 2001, La Habana, Cuba.

Cabero Almenara, J.: Las posibilidades de las nuevas tecnologías de la información y la comunicación para los desafíos de la educación de las personas adultas. En: www.ugr.es/ sevimeco.documentos/iteoricas/it06d.htm

Cebrián De La Serna, M. (ed.). (2000). Campus virtuales y enseñanza universitaria. Málaga, Universidad de Málaga.

García Aretio, L. (2001). La educación a distancia. De la teoría a la práctica. Barcelona: Ariel.

Gutiérrez Pérez, F.; Prieto Castillo, D. (1999). La mediación pedagógica. Apuntes para una educación a distancia alternativa. 6a. ed. Buenos Aires: Ciccus / La Crujía.

LiBEDINSKY, M. (1999). Tres recomendaciones para diseñadores didácticos de materiales para proyectos de educación a distancia. En: www.bitacora.org/articulos/arti-05.htm.

LibedinsKy, M. (1999). El módulo no es un resumen; es una brújula. En: www.bitacora.org /articulos/arti-01.htm.

Mansilla Gómez, G. Y Casas Raposo, I. (2000). Una metodología de evaluación de sistemas de educación interactiva a distancia basados en web. Viña del Mar (Chile), V Congreso RIBIE 2000. En file://H:papers\287index.html

Martín Rodríguez, E. Y AhiJado Quintillán, M. (corrd.). (1999). La educación a distancia en tiempos de cambios: nuevas generaciones, viejos conflictos. Madrid: De la Torre.

Nielsen, J. (2000). Usabilidad. Diseño de sitios web. Madrid: Pearson Educación / Prentice Hall.

Padula Perkins, J. (2001). La capacitación profesional a distancia en el área de la salud. En www.bitacora.org/opinion.php3.

Perales Palacios F. (dir.). (2000). Didáctica de las ciencias experimentales. España: Marfil.

Rey VALZACCHI, J. (1998) Internet y educación. Aprendiendo y enseñando en los espacios virtuales. Buenos Aires: Horizonte Informática Educativa.

Silvio, J. (2000). La virtualización de la universidad. Caracas: IESALC/UNESCO.

Tiffin, J. Y Rajasingham, L. (1997). En busca de la clase virtual. Barcelona: Paidós.

UÑANTES G.; REYNOSO E.; BRESCIA M. (2001). E-learning: cambiando paradigmas en capacitación. En www.bitacora.org/opinion.php3?hoy=2001-10-25.

Villafuerte, O. (1999). Guía para el diseño web. En: CD Ware 53(marzo), 56-66. 
Resultados de la investigación evaluativa de un curso a distancia de genética humana para profesionales...

\section{PERFIL ACADÉMICO Y PROFESIONAL DE LOS AUTORES}

Dr. Plácido Daniel Blanzaco. Profesor Titular Ordinario y Director del Departamento de Bioquímica Clínica y Cuantitativa de la Facultad de Bioquímica y Cs. Biológicas de la Universidad Nacional del Litoral. Director del Departamento de Educación a Distancia del Colegio de Bioquímicos de Entre Ríos. Docente investigador. Sus líneas de trabajo son la educación a distancia y los multimedios orientados a las ciencias de la salud.

Dirección: Monte Caseros 342 -3100-Paraná-Entre Ríos-República Argentina

TE: +54 (343) 4220072 - Fax: +54 (343) 4231538

E-mail: pdblanzaco@infovia.com.ar

MSc. Cecilia María Brissón. Profesor Adjunto Ordinario del Departamento de Bioquímica Clínica y Cuantitativa de la Facultad de Bioquímica y Cs. Biológicas de la Universidad Nacional del Litoral. Vicedirector del Departamento de Educación a Distancia del Colegio de Bioquímicos de Entre Ríos. Miembro de la Sociedad Argentina de Investigación y Educación Médica (SAIDEM). Docente investigador. Sus líneas de trabajo son los multimedios y la educación a distancia orientados a las ciencias de la salud.

Dirección: Roca 181 -2820-Gualeguaychú-Entre Ríos-República Argentina

TE: +54 (3446) 435245

E-mail: cbrisson@entrerios.net

Lic. Roberto Ronchi. Licenciado en Ciencias de la Educación. Profesor Titular. Docente investigador en la Facultad de Ingeniería de la Universidad Nacional de Entre Ríos. Sus líneas de trabajo son los multimedios, la tecnología en la educación y la educación a distancia; se ocupa además de la metodología y la divulgación en la enseñanza de las ciencias.

Dirección: Las Golondrinas 354 - 3100 - Oro Verde - Entre Ríos - República Argentina.

TE +54 (343) 4975049 .

E-mail: rronchi@gamma.com.ar

Bioq. Angela María Pedro. Jefe de Trabajos Prácticos Ordinario del Departamento de Bioquímica Clínica y Cuantitativa de la Facultad de Bioquímica y Cs. Biológicas de la Universidad Nacional del Litoral. Docente investigador. Sus líneas de trabajo son los multimedios y la educación a distancia orientados a las ciencias de la salud.
Dirección: Simón de Iriondo 1306 -3000-Recreo-Santa Fe-República Argentina
TE: +54 (342) 4960229
E-mail: hodetti@fbcb.unl.edu.ar

Departamento de Bioquímica Clínica y Cuantitativa de la Facultad de Bioquímica y Cs. Biológicas de la Universidad Nacional del Litoral - Colegio de Bioquímicos de Entre Ríos

E-mail: pdblanzaco@infovia.com.ar

Facultad de Bioquímica y Cs. Biológicas de la Universidad Nacional del Litoral

CC 242 - Paraje El Pozo - Ciudad Universitaria

Tel.: +54 (342) 4575-206/209/215/216/217 - Fax: +54 (342) 4575-221

3000 - Santa Fe - Provincia de Santa Fe - REPUBLICA ARGENTINA

Colegio de Bioquímicos de Entre Ríos

España 234

Tel-Fax: +54 (343) 4318110

3100 - Paraná - Provincia de Entre Ríos - REPUBLICA ARGENTINA 